\title{
CFD Technique for Solving Low Water Level Problem of Axial Flow Pumps
}

\author{
Sami Abdel-Fattah Abdel-Ghani El-Shaikh \\ Mechanical and Electrical Research Institute, National Water Research Center, Cairo, Egypt
}

Email address:

samiabd@yahoo.com

\section{To cite this article:}

Sami Abdel-Fattah Abdel-Ghani El-Shaikh. CFD Technique for Solving Low Water Level Problem of Axial Flow Pumps. American Journal of Water Science and Engineering. Vol. 3, No. 3, 2017, pp. 34-44. doi: 10.11648/j.ajwse.20170303.11

Received: July 5, 2017; Accepted: July 21, 2017; Published: September 12, 2017

\begin{abstract}
In this paper, water levels variation in the sump intake is studied in El- SHABAB Pumping Station. ANSYS Ver.17.1 flow simulation software is used to simulate the flow conditions to overcome decreasing water level in the low demands period, winter closure. Seven scenarios are studied to obtain the best one to apply it at that condition. Case 1 represents the optimum design when the submergence water level is $5 \mathrm{~m}$ above the sump floor and the length of submersed part in the sump is $3.5 \mathrm{~m}$. Case 2 represents the winter closure period where the submergence water level decreased by $1.5 \mathrm{~m}$ to become $3.5 \mathrm{~m}$ in the period winter closure and the length of submersed part in the sump is $2 \mathrm{~m}$. As a result, velocity in the suction pipe decreased $26 \%$, a swirl angle $10.79^{\circ}$ is attained, and vibration level increased 4 times representing hydraulic and dynamic problems. Case 3 is done by adding a joint with length $0.5 \mathrm{~m}$ to the submersed part of pipe. Case 4 is done by increasing a joint length by $1 \mathrm{~m}$ to the submersed part of pipe. Case 5 is done by adding a cone under the bell mouse with height $1 \mathrm{~m}$ and with the same pipe diameter. Case 6 is done by adding a cone under the bell mouse with height $1 \mathrm{~m}$ and $0.5 \mathrm{~m}$ joint leading to increase the submersed part of pipe to become $2.5 \mathrm{~m}$. Case 7 is done by repeating case 6 with increasing water submerging level after finishing the period of winter closure where the water level in the sump is $5 \mathrm{~m}$. The results indicate that, during the period of winter closure, Case 6 is the best condition to use where it prevents vortex and has the best swirling angle $5.12^{\circ}$ with no turbulence is observed at the entrance of the pump improving the dynamic and hydraulic performance of the pump. Also, the results indicate that Case 7 after finishing the period of winter closure and water level returned to become $5 \mathrm{~m}$ (optimum design) is the best condition to use during all seasons where it prevents vortex and has the best swirling angle $4.66^{\circ}$.
\end{abstract}

Keywords: Pump Intake, Vortex, Computational Fluid Dynamics (CFD), Hydraulic Problems, Suction Sump, Water Level, Simulation

\section{Introduction}

Pump performance curves provided by the pump manufacture are obtained on the condition of no vortices flowing into the pump intake and also dynamic analysis was done. A water suction level in the intake of pump stations is the most important factors affecting the efficiency of those stations. Therefore, it is necessary to determine the best water level in the inlet channel, without affecting the efficiency of the units and avoid hydraulic and dynamic problems. The flow conditions at pump entry depend upon flow conditions in approach channel, sump geometry, location of pump intake with respect to the walls, velocity changes, obstructions such as piers, screens, and rotational tendencies in flow produced upstream of the pump bays [1]. A pump intake was modified to eliminate air entrainment. An experimental model was built with these modifications, where the air entrainment was seen to be eliminated [2]. A swirling flow occurs when pump-approach flow distributions within the intake bay are not uniform regardless of its origin. These problems encountered in the pump intake will affect the pump performance and significantly increase the operational and maintenance costs. Large amount of energy is consuming in pumping station for water supply. In addition to hydraulic features, such as the head and discharge of pumped water, geometric design of pumping could affect the amount of essential energy [3]. In modeling flow in a pump sump to evaluate the potential for the formation of vortices, the geometric scale is selected to minimize viscous and surface 
tension scale effects [4]. Several modifications to the initial design were developed in the model to improve the lateral distribution of flow within circulating water pump bays. Reducing the vortex activity in the vicinity of the pump such that the hydraulic conditions in the sump would meet the specified performance criteria [5]. Zaho et al [6] studied the flow characteristics of pump sump and performance analysis of the mixed flow pump. The efficiency of anti-vortex devices to insure the uniform flow is confirmed. From the numerical analysis, the inception of cavitation is observed on the suction surface where the leading edges meet the tip, and then the cavitation zone expands. Rajendran et al [7] have made numerical analysis for the flow characteristics of a sump model with pump intake and good agreements were achieved by comparing the numerical results with the experiments. Iwano et al [8] have introduced a numerical method for the submerged vortex by analyzing the flow in the pump sump with and without baffle plates. Lee et al [9] have conducted the CFD analysis of a multi-intake pump sump model to check the flow uniformity by predicting the location, number and vorticity of the vortex. Lee [10] has suggested a design guideline for the shape of pump sump by model test and CFD analysis. Moreover, Turbomachinery Society of Japan [11] has revised the standard of pump sump model test and the revised standard examined the possibility of numerical analysis for the prediction of the flow in the sump model using several commercial and in-house CFD codes. Kim et al [12] examined the flow uniformity according to the flow distribution in the pump intake channel to find out the cause of vortex occurrence in detail by experiment and CFD analyses. The main concern and challenge in this study is to investigate the effect of low suction water levels on the hydraulic performance of axial flow pumping stations. Effectiveness of suitable flow rate is determined under variation of the water suction levels.

\section{Problem Description \& Task}

In this research, a numerical solution for solving the problems that occurs at low suction water levels during the low demands period (winter closure) using CFD technique at El- SHABAB Pumping Station. The flow conditions at pump entrance are affected by sump design and its associated pumping and entrance conditions. Hydraulic measurements are done for the axial flow pumping units. The water levels in El-Ismailia canal is varied from low levels at winter (closure period) to the maximum water levels at the summer season due to the maximum water requirements. This variation of water levels in El-Ismailia canal affects the performance of pumping stations. The main task of this paper is to evaluate the hydraulic performance of the axial flow pumping stations when operating at low suction levels and evaluate the availability of this pumping station using CFD ANSYS software Ver. 17.1. The software solves the Navier-Stock equations, which are formulations of mass, momentum, and energy conservation lows for liquid flow [13], the continuity equation (1) and conservation of momentum (2) can be written as follows:

$$
\begin{gathered}
\nabla \rho u_{r}=0 \\
\nabla \rho u_{r}+2 \rho \Omega * u_{r}+\rho \Omega * \Omega * r=-\nabla P+\mu_{e f f} \nabla^{2} u_{r}
\end{gathered}
$$

Where:

$\rho$ is the density of the fluid, \& $p$ is the static pressure,

$u_{r}$ is the vector fluid velocity in the rotating system, $\& \Omega$ is the rotational speed and

$\mu_{e f f}$ is the dynamic effective viscosity.

The equations are supplemented by fluid state equations defining the nature of the fluid, and by empirical dependencies of fluid density, viscosity and thermal conductivity on temperature. To predict turbulent flows, the Favre-averaged Navier-Stokes equations are used, where time-averaged effects of the flow turbulence on the flow parameters are considered. Large-scale, time-dependent phenomena are taken into account directly. El- SHABAB Pumping Station located at the area of El-SALHIYA. It's used to serve irrigation of 9500 feddans and consists of 6 pump units; each pump unit is of discharge $1.5 \mathrm{~m} 3 / \mathrm{sec}$, head $50 \mathrm{~m}, 985 \mathrm{rpm}$, and motor power $1000 \mathrm{~kW}$, as shown in Figure 1-a. The pumping station receives the feed water from El-Ismailia main canal, as shown in Figure 1-b.

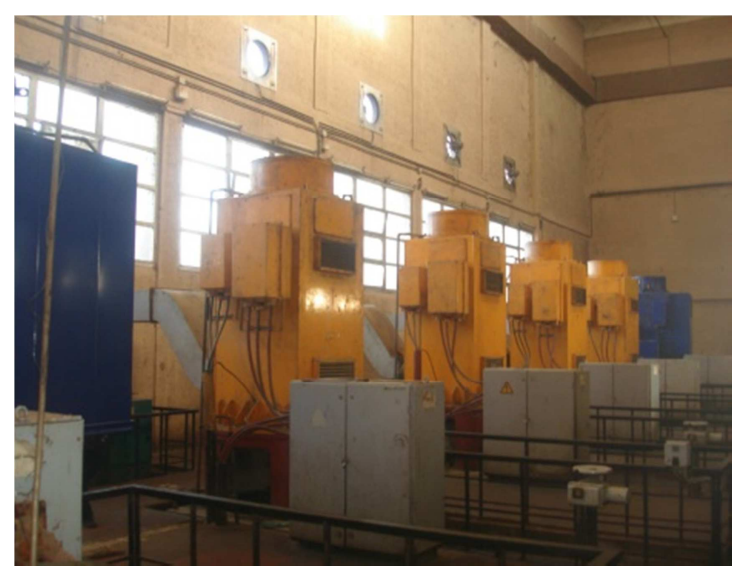

(a) El- SHABAB pumping station

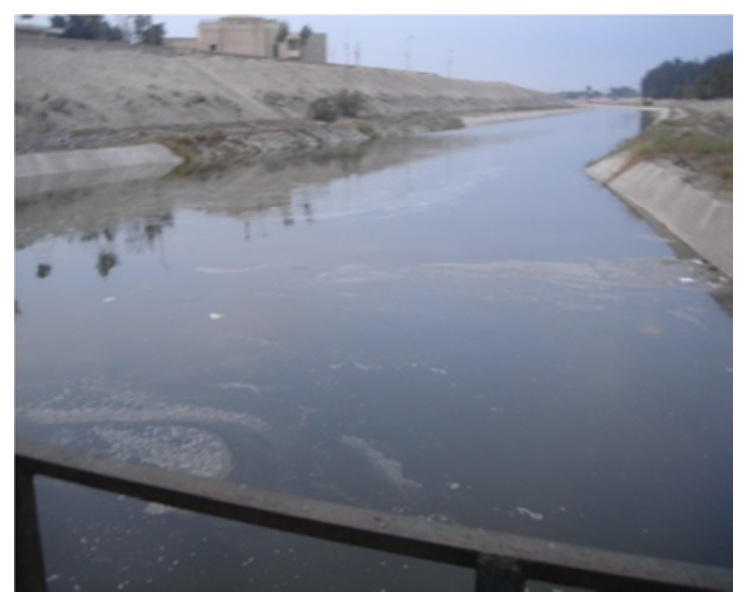

(b) Photographs of Pump Intake

Figure 1. El-SHABAB (1) Pumping Station. 


\section{Results of Hydraulic Measurements Tests}

Hydraulic performance tests are done during working operating range, where the control valve in the delivery pipe is used to control flow rate, as shown in Table 1-a. The actual operation point measured at pump speed, $305 \mathrm{rpm}$. The measured flow rate is higher than the design values by $8.66 \%$; this increase in flow arte due to the total dynamic head is less than the design value $(50 \mathrm{~m})$. The water level at this point is about $4.85 \mathrm{~m}$. On the other hand, when start pumps number two there are excess vibration and hydraulic problems appears. However, the flow details, which could not be experimentally obtained inside the pump, are numerically obtained. In the low demands period (winter closer), the water level at sump is decreased to $3.6 \mathrm{~m}$, the measured flow rate reach to $1.53 \mathrm{~m}^{3} / \mathrm{s}$ as shown in Table $1-b$ and Figure 2. At the design flow arte the pump power consumption increases by $4 \%$, this increase in the power consumption due to increase in lifting head. The overall efficiency is decreased about $5 \%$ also.

Table 1 Hydraulic measurements at maximum water level $(4.85 \mathrm{~m})$ and decreasing water level to $3.6 \mathrm{~m}$.

Table 1-a. Hydraulic measurements when sump water level $5 \mathrm{~m}$.

\begin{tabular}{lllll}
\hline $\mathbf{Q}\left(\mathbf{m}^{3} / \mathbf{s e c}\right)$ & Total Head $(\mathbf{m})$ & Water Power $(\mathbf{k W})$ & Electric Power $(\mathbf{k W})$ & Overall Eff $(\mathbf{\%})$ \\
\hline 1.63 & 35.00 & 559.39 & 910 & 61.71 \\
1.5 & 38.87 & 571.59 & 930 & 65.81 \\
1.35 & 43.08 & 570.21 & 933 & 60.50 \\
1.25 & 45.54 & 558.10 & 920 & 57.85 \\
1.15 & 46.59 & 525.31 & 900 & 58.08 \\
\hline
\end{tabular}

Table 1-b. Hydraulic measurements when sump water level $3.5 \mathrm{~m}$.

\begin{tabular}{lllll}
\hline $\mathbf{Q}\left(\mathbf{m}^{3} / \mathbf{s e c}\right)$ & Total Head $(\mathbf{m})$ & Water Power $(\mathbf{k W})$ & Electric Power $(\mathbf{k W})$ & Overall Eff $(\mathbf{\%})$ \\
\hline 1.53 & 39.16 & 587.38 & 967 & 60.74 \\
1.42 & 39.72 & 552.91 & 956 & 57.84 \\
1.31 & 44.28 & 568.75 & 951 & 59.81 \\
1.18 & 47.09 & 544.81 & 944 & 57.71 \\
1.05 & 47.79 & 491.98 & 935 & 52.62 \\
\hline
\end{tabular}

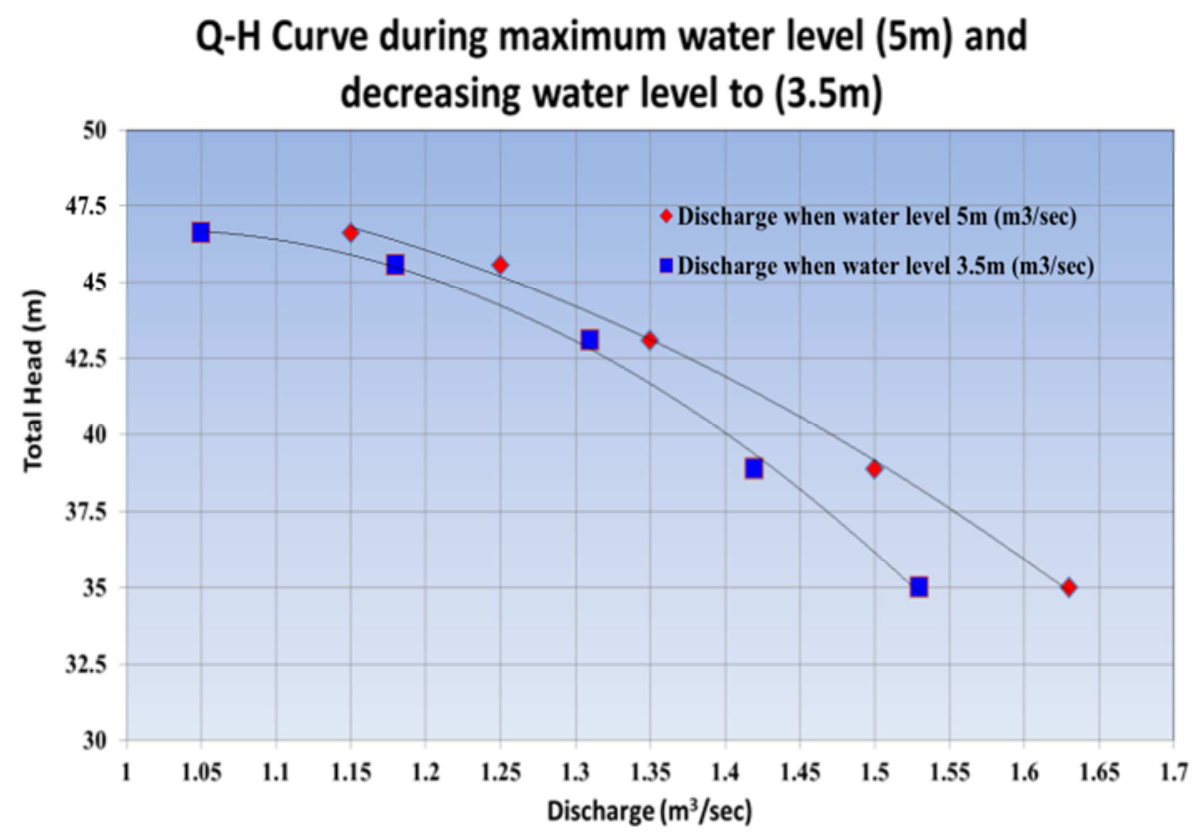

Figure 2. Pump performance curve for El-SHABAB (1) Pumping Station at level 3.6m.

\section{Results of Vibration Measurements Tests}

Overall vibration levels were measured in terms of root mean square velocity to evaluate dynamic condition of the rotating parts according to the standards [ISO10816-1,1995] [14]. Also, vibration spectra were done to determine the vibration sources, exciting frequencies, and dynamic conditions of the different components of the pumping station. Results of measurements done on different components of the pumping station are shown in Table 2. Vibration were measured and analyzed on the pumping station on the motor non drive end, on the motor drive end, sleeve, Pump bearing, pump casing, and delivery pipe, as shown in Figure 3. 


\subsection{Overall Vibration Levels}

Results of measurements show that the overall vibration levels measured at low suction water levels at winter closure period is higher $400 \%$ than that was measured in case of normal water level $(5 \mathrm{~m})$. Vibration levels at other components are more than $200 \%$ of the corresponding parts at winter closure period, as shown in Table [2].

\subsection{Dynamic Analysis}

Figure 4-a, shows vibration velocity spectrum measured on the motor non drive end when water level is $(5 \mathrm{~m})$. Vibration velocity spectrum indicates that there are no high peaks meaning there are no any mechanical or hydraulic problems. Figure 4-b, shows vibration velocity spectrum measured on the motor non drive end when water level decreased to become $(3.5 \mathrm{~m})$. Vibration velocity spectrum shows vibration peaks at the motor running speed $(16 \mathrm{~Hz})$ and its second and third harmonics indicating mechanical problems. Vibration level measured at the running speed is $9.2 \mathrm{~mm} / \mathrm{sec}$, and of order $2.5 \mathrm{~mm} / \mathrm{sec}$ at its higher harmonics. These mechanical problems contributed to damaging to motor-drive-end bearing, which is diagnosed based on the bearing elements frequencies $180 \mathrm{~Hz}$ and 350 $\mathrm{Hz}$ of vibration level $3 \mathrm{~mm} / \mathrm{s}$. It can be concluded that the diagnosed mechanical problems for the motor is the source of high vibration level subjecting the structure and human to risk and danger.

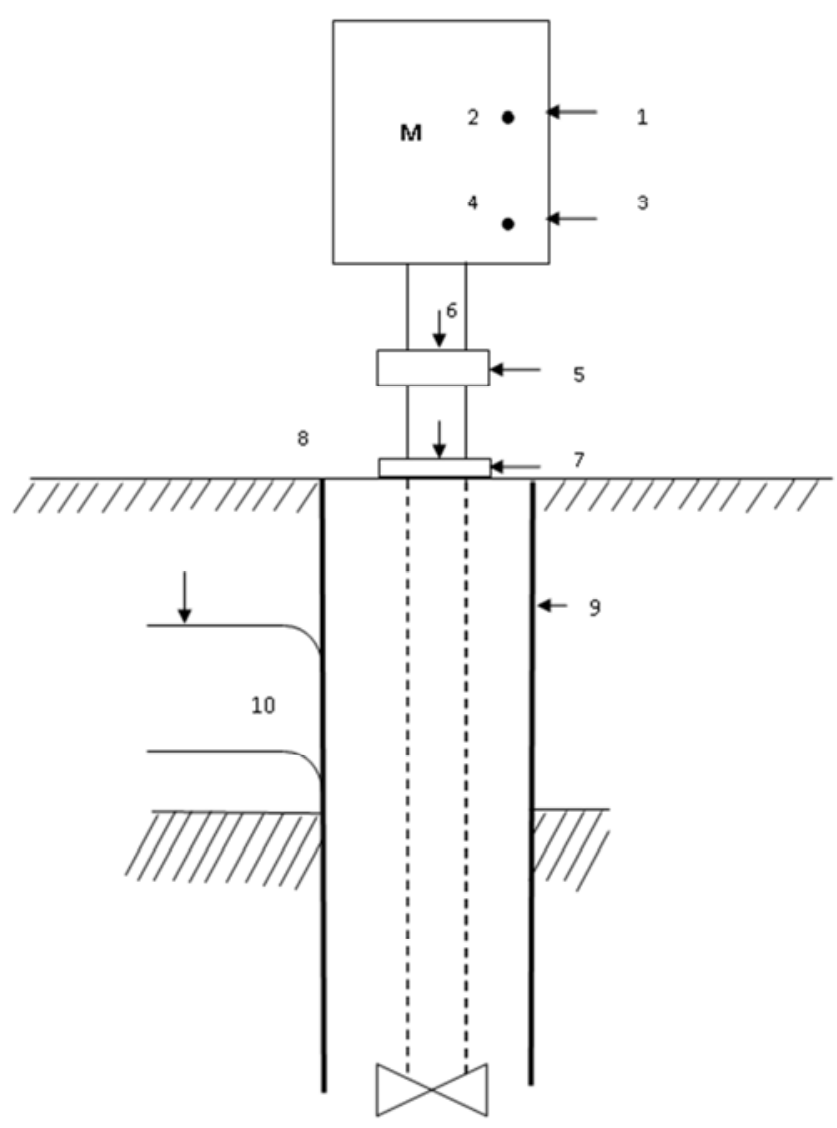

Figure 3. Location measurements.
Table 2. The overall vibration levels measured at various suction water levels.

\begin{tabular}{lll}
\hline $\begin{array}{l}\text { Point } \\
\text { No }\end{array}$ & Locations & $\begin{array}{l}\text { Overall Vibration } \\
\text { Level }(\mathbf{m m} / \mathbf{s})\end{array}$ \\
\hline 1 & Motor non drive end Radial & 12.67 \\
2 & Motor non drive end Radial Perpendicular & 4.676 \\
3 & Motor drive end Radial & 6.937 \\
4 & Motor drive end Radial Perpendicular & 2.885 \\
5 & Sleeve Axial & 7.279 \\
6 & Sleeve Radial & 2.322 \\
7 & Pump bearing Axial & 0.826 \\
8 & Pump bearing Radial & 6.239 \\
9 & Pump Casing Radial & 2.024 \\
10 & Delivery Pipe Radial & 1.714 \\
\hline
\end{tabular}

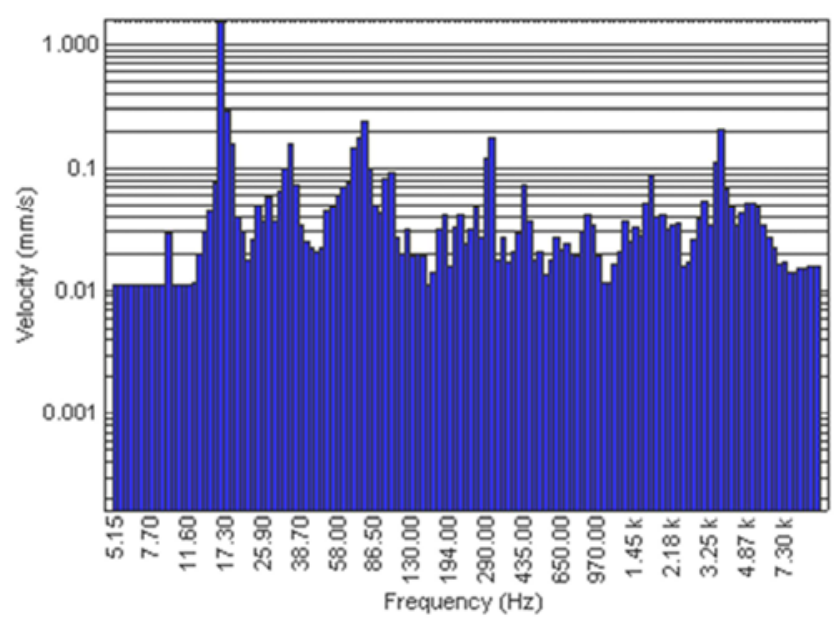

(a) Vibration Velocity spectrum measured on the Motor non drive end during Water Level $(5 \mathrm{~m})$

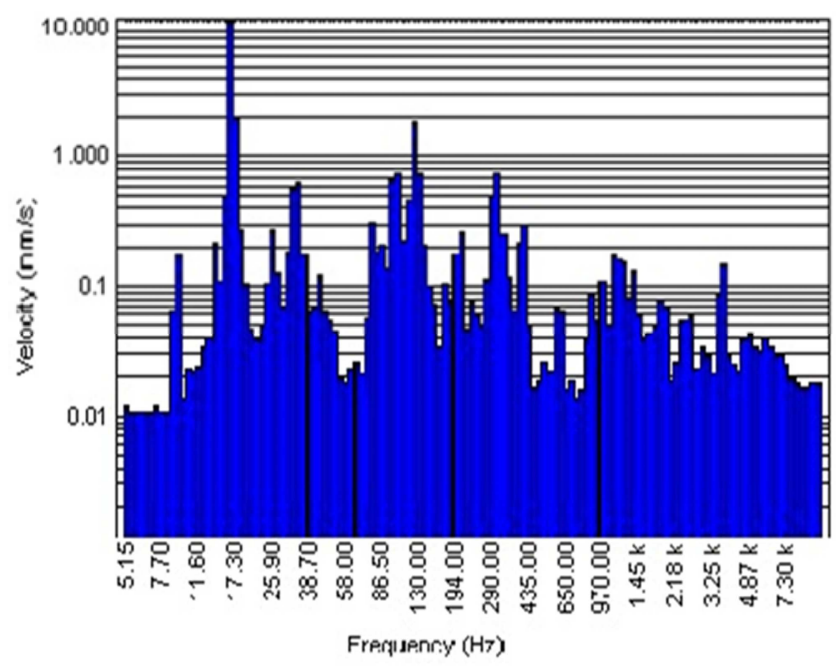

(b) Vibration Velocity spectrum measured on the Motor non drive end during Water Level $(3.5 \mathrm{~m})$

Figure 4. Vibration spectra velocity spectrum measured on the Motor non drive end during maximum water level $(5 \mathrm{~m})$ and during decreasing water level $(3.5 \mathrm{~m})$.

\section{Numerical Studies}

The quality of the computational mesh has an important role in achieving the desired accuracy of the simulations especially if the computational domain is very complex. The basic three- 
dimensional geometry is prepared using Fluent under Ansys Ver.14.5 flow simulation software drawing with the grid surfaces plot of sump. The equations are supplemented by fluid state equations defining the nature of the fluid, and by empirical dependencies of fluid density, viscosity and thermal conductivity on temperature. To predict turbulent flows, the Favre-averaged Navier-Stokes equations are used, where time-averaged effects of the flow turbulence on the flow parameters are considered, where the other, i.e. large-scale, time-dependent phenomena are taken into account directly. Three dimensional unstructured meshes are used for the flow simulation in the pump sump. Unstructured mesh is used for this study due to model complexity and easy to be mesh especially at the intake section. The numerical solver uses unstructured meshes which allow flexibility in meshing very complex geometries while maintaining high quality computational mesh which is necessary for obtaining accurate solutions [13].

\subsection{Geometry and Grid Generation}

Three-dimensional geometry for pump station sump using ANSYS Modeler was done. The meshes required for the calculations were generated and checked by using the ANSYS Fluent preprocessing package. Unstructured tetrahedral mesh, using ANSYS mesh modeler, is used. EquiAngle Skew and EquiSize Skew (parameter were that used for mesh accuracy must be less than less than 0.9) of the grid were all of them were less than 0.95 , so the grid quality is good. Figure (5-a, 5-b) shows the 3D model and wall grid of the calculation region. The model has 400000 nodes with tetrahedral mesh.

\subsection{Boundary Conditions}

In the present study, mass flow inlet and outflow boundary conditions were used for the inlet and outlet respectively. Outer walls were stationary, no flow can pass and the velocity at the surface is zero non slip boundary conditions. The turbulence intensity at the inlet totally depends on the upstream history of flow. Since the fluid in the suction tank is undisturbed, the turbulence intensity for all conditions is considered $5 \%$. Water was used as a working fluid in ambient condition.

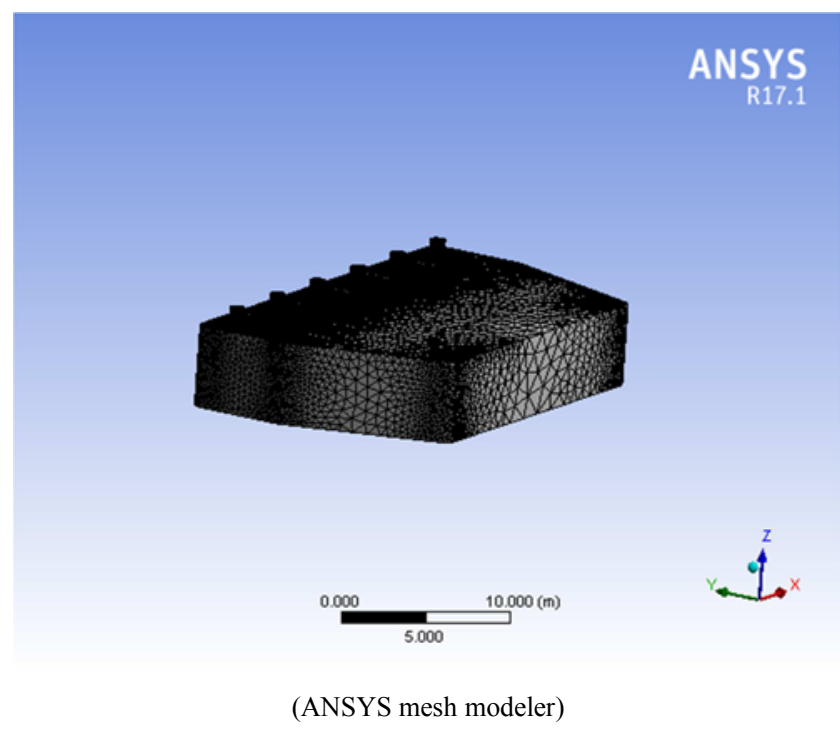

Figure 5a. The 3D wall grid mesh The surface and the volume mesh of the sump intake.

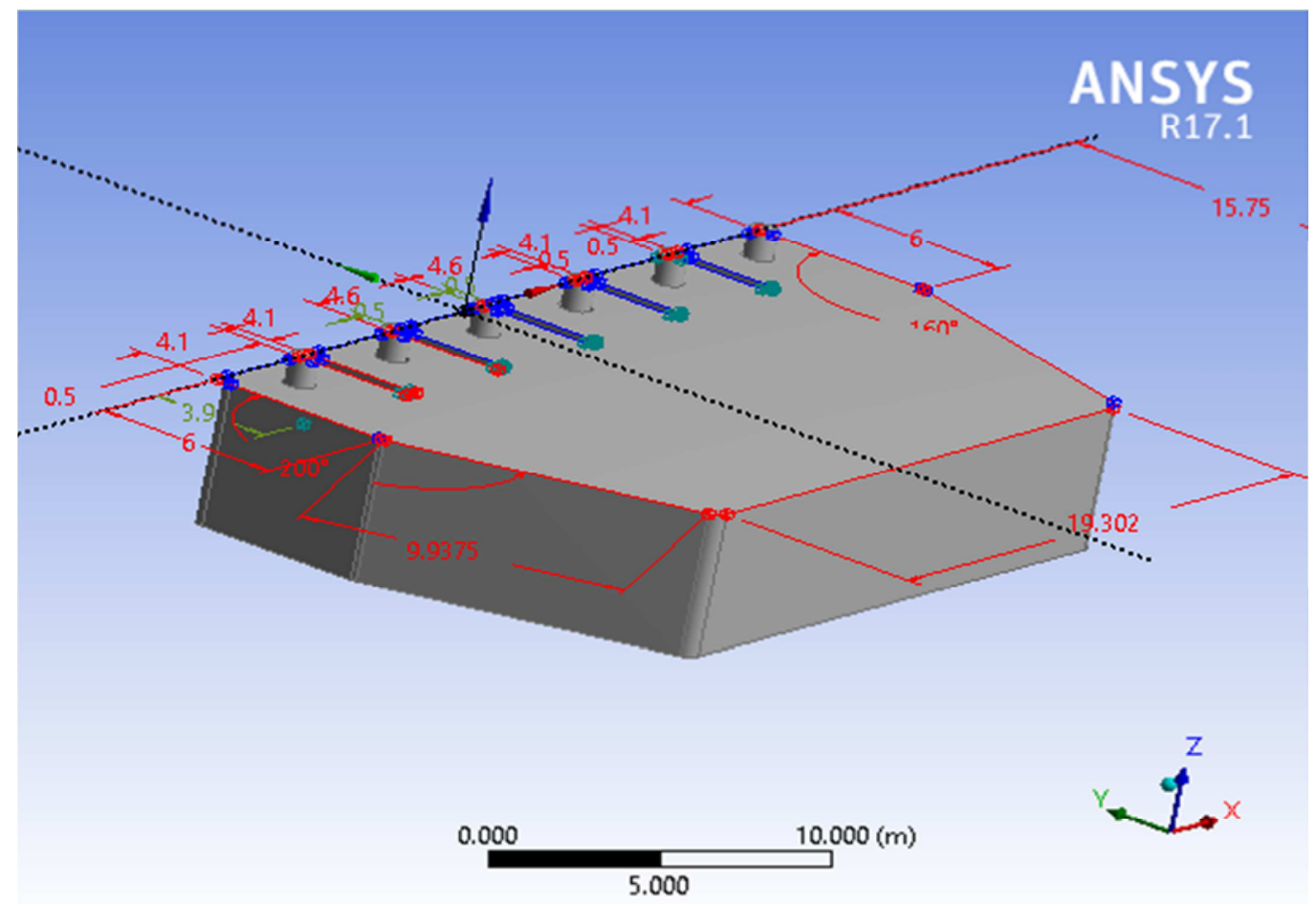

(ANSYS Modeler)

Figure 5b. The 3-D model Profile views of the pumping station. 


\subsection{Solver}

ANSYS Fluent 17.1 was used to simulate the inner flow field under non cavitation condition. The standard k- $\varepsilon$ turbulence model and simple algorithm are applied to solve the equations. The simulation is steady and Convergence precision of residuals is $10^{-6}$.

\subsection{Swirl Angle}

It is essential to ensure that the pumps operating in such pump intakes get smooth swirl free flow at their inlets. Proper intake design provides uniform swirl free flow to the pumps and swirl angle types as shown in equations below. Undesirable flow conditions could be eliminated / reduced to minimum, by incorporating the necessary modifications in sump and their effectiveness could be ascertained by studying the flow under modified conditions. The model test results is used to determine if the initial design of the pump sump structure would be susceptible to surface and subsurface vortices, pre-swirl entering pumps and excessive fluctuation in the velocities at the pump bell throat according to Hydraulic Institute (HI) specifications. The results of the tests showed that the rotations of the roto-meters are within the allowable limits specified by the $\mathrm{HI}$ criteria in all operation modes.

Where,

$$
\begin{gathered}
\theta=\tan ^{-1} \frac{V_{R}}{V_{A}} \\
V_{R}=\frac{\pi d_{v} R}{60} \\
V_{A}=\frac{Q}{A}
\end{gathered}
$$

Where,

$\mathrm{V}_{\mathrm{R}}=$ Rotational velocity $(\mathrm{m} / \mathrm{s})$

$\mathrm{V}_{\mathrm{A}}=$ Axial velocity $(\mathrm{m} / \mathrm{s})$

$\mathrm{D}_{\mathrm{v}}=$ Diameter of voltammeter vane $(\mathrm{m})$

$\mathrm{R}=$ Number of revolutions of the vane per minute (Rev/min)

$\mathrm{Q}=$ Flow through the pump intake $\left(\mathrm{m}^{3} / \mathrm{s}\right)$

$\mathrm{A}=$ Cross-sectional area of the intake $\left(\mathrm{m}^{2}\right)$

\section{Results \& Discussions}

\subsection{Case One: Original Case (Optimum Design)}

In this case, flow measurement is $1.5 \mathrm{~m}^{3} / \mathrm{sec}$, water level is $5 \mathrm{~m}$ (submergence level) above the sump floor, length of submergence pipe in the sump is $3.5 \mathrm{~m}$, and four units are operating. This case is considered the standard case of pumping station. The model results show that; the velocity has a good distribution, there is no effect of any vortices under the submersed pipes face, and velocity is in the range of $0.08 \mathrm{~m} / \mathrm{sec}$ and this value is the ideal velocity speed in the intake, as shown in stream line velocity Figure (6-a). Also, Velocity ranged from $2.708 \mathrm{~m} / \mathrm{sec}$ to $3.385 \mathrm{~m} / \mathrm{sec}$ inside suction pipes and this value is the ideal velocity speed in the submergence pipe according to this pipe diameter (ANSI 98 [15]), as shown in velocity Contour Figure (6-b). This case occurs when the submersed part of pipe is $3.5 \mathrm{~m}$. Where, the standard [9] indicates that the inlet velocity mustn't increase than $0.3 \mathrm{~m} / \mathrm{sec}$ at the input of the intake of the suction pipe and velocity in the intake pipe in the range from $2 \mathrm{~m} / \mathrm{sec}$ to 5 $\mathrm{m} / \mathrm{sec}$. The swirl angle in this case is about $4.85^{\circ}$ and this is in the acceptable limit where it is less than $5^{\circ}$ (As suggested in $\mathrm{HI})$ [16].

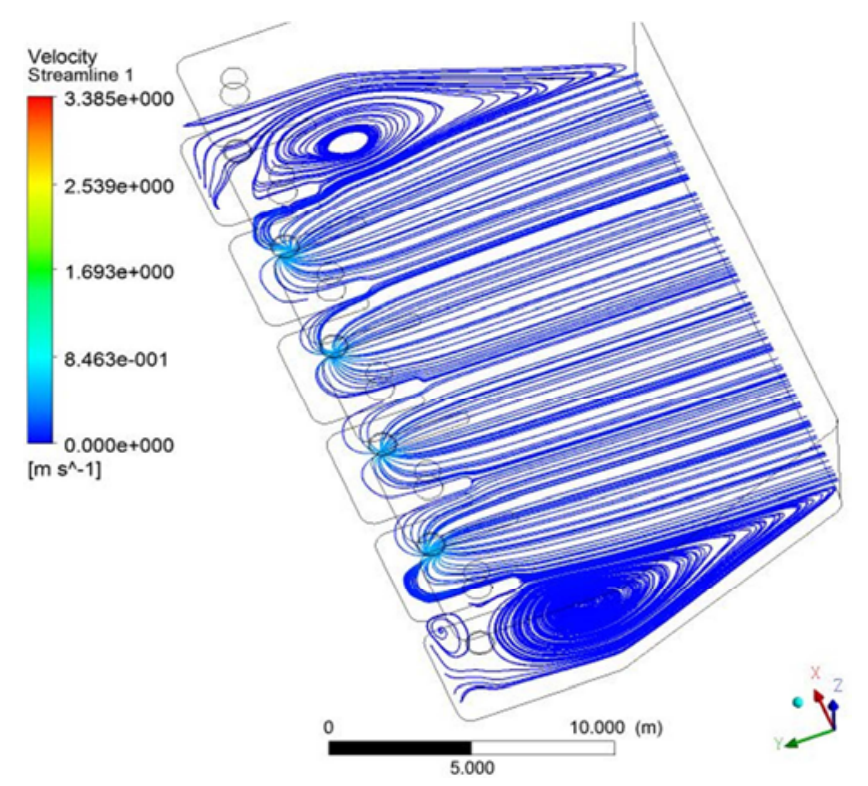

(a) Stream Line Velocity
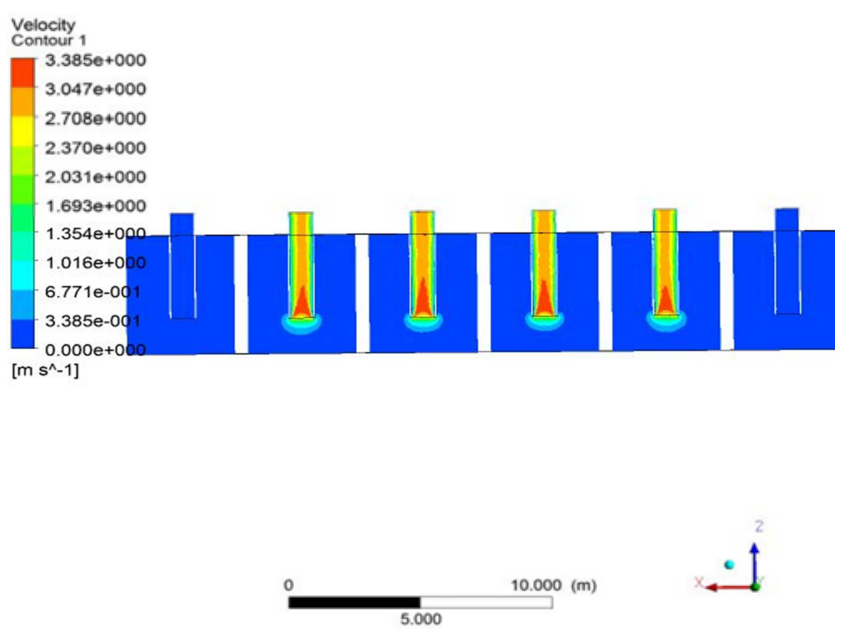

(b) Velocity Contour (distribution)

Figure 6. Case One: Original Case (Optimum Design).

\subsection{Case Two: Reducing Water Submerging Level to Be $3.5 m$}

In this case, reducing water submerging level to be $3.5 \mathrm{~m}$ from the ground due to winter closing where the submersed 
pipe decreased by $1.5 \mathrm{~m}$ to become $2 \mathrm{~m}$ instead of $3.5 \mathrm{~m}$, and four units are operating. The model results show; the effect of vortices under the suction surface is apparent, the velocity vector has small disturbance in the inlet of submersed pipe, as shown in stream line velocity Figure (7-a). Velocity inside the suction pipes decreases to value from $1.857 \mathrm{~m} / \mathrm{sec}$ to $2.321 \mathrm{~m} / \mathrm{sec}$ as shown in velocity contour Figure (7-b). The results of this case indicate that the effect of vortices occurs in all pipes. In this case after reducing water submerging Level to become $3.5 \mathrm{~m}$ from ground and from calculating, the swirl angle is $10.79^{\circ}$ and this is much higher than the acceptable limit, where the acceptable limit mustn't greater than $5^{\circ}$ (As suggested in HI) [16]. Hence sump geometry is not adequate for safe operation of pumps and sump. Thus for efficient and safe operation of pumps and sump, it is required to modify the sump geometry. It was observed that vortices of type 3 as shown in Figure (7-a).

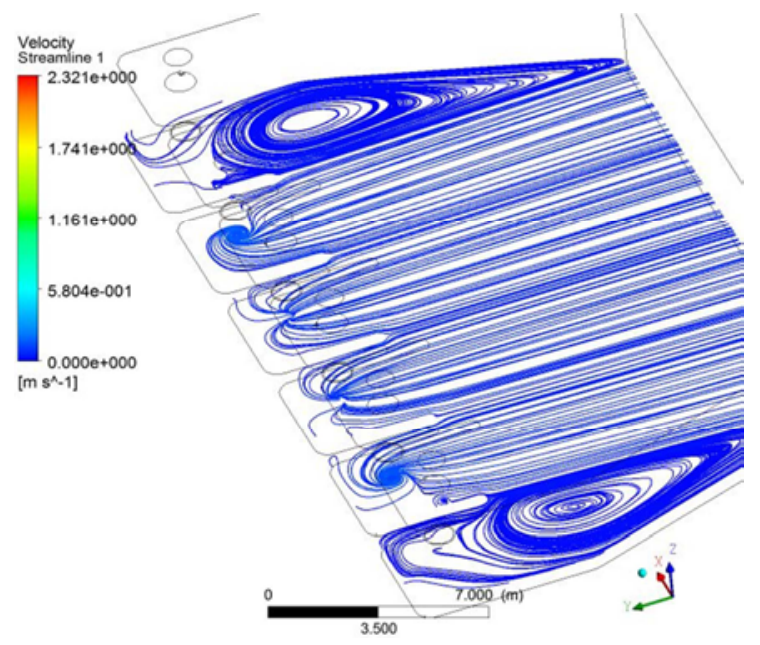

(a) Stream Line Velocity

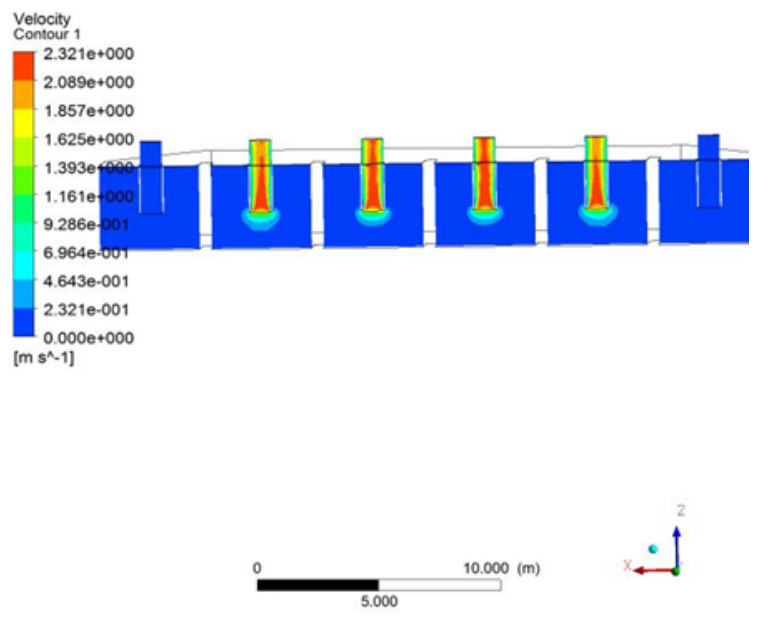

(b) Velocity Contour (distribution)

Figure 7. Case Two: Reduce water submerging Level to become $3.5 \mathrm{~m}$ (from ground).

\subsection{Case Three: Adding Joint with Length $0.5 \mathrm{~m}$}

In this case, adding a joint with length $0.5 \mathrm{~m}$, this means increased the submerging pipe by $0.5 \mathrm{~m}$ leading to increase the length of submersed part of pipe to become $2.5 \mathrm{~m}$, and four units are operating in this case. The model results show that the vortices disappear from all suction pipes and decreasing disturbance at the submersed pipe inlet is occurred better than case before as shown in stream line velocity Figure (8-a). Velocity value inside the suction pipes increased better than case before where it ranged from 1.858 $\mathrm{m} / \mathrm{sec}$ to $2.322 \mathrm{~m} / \mathrm{sec}$ as shown in velocity contour Figure (8b). The results of this case indicate that the flow become uniform better than case before, and the effect of vortices were disappeared from the intake and in all units and all submerging pipe become fully filled with water leading to increase total efficiency. In this case, the calculated swirl angle is slightly decreased than case 3 where it reached to $7.05^{\circ}$ but this angle still higher than acceptable limit (As suggested in $\mathrm{HI}$ ). It was observed that vortices of type 1 as shown in Figure (8-b).

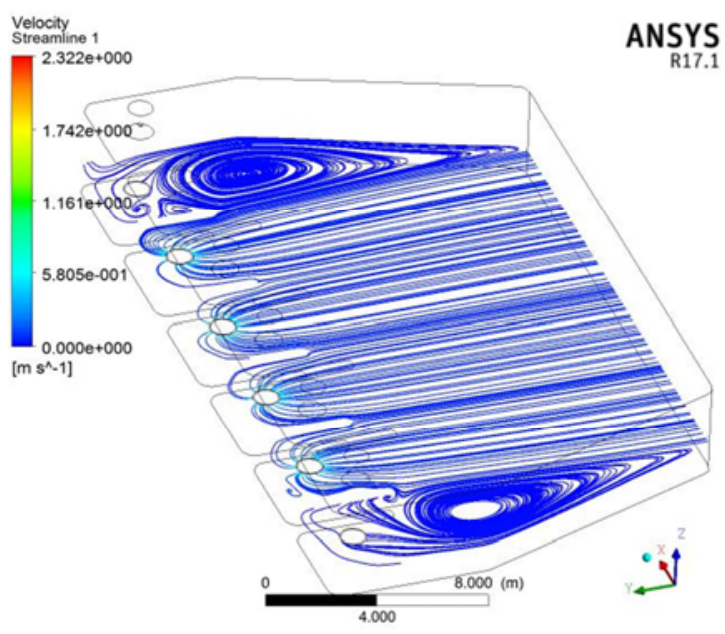

(a) Stream Line Velocity
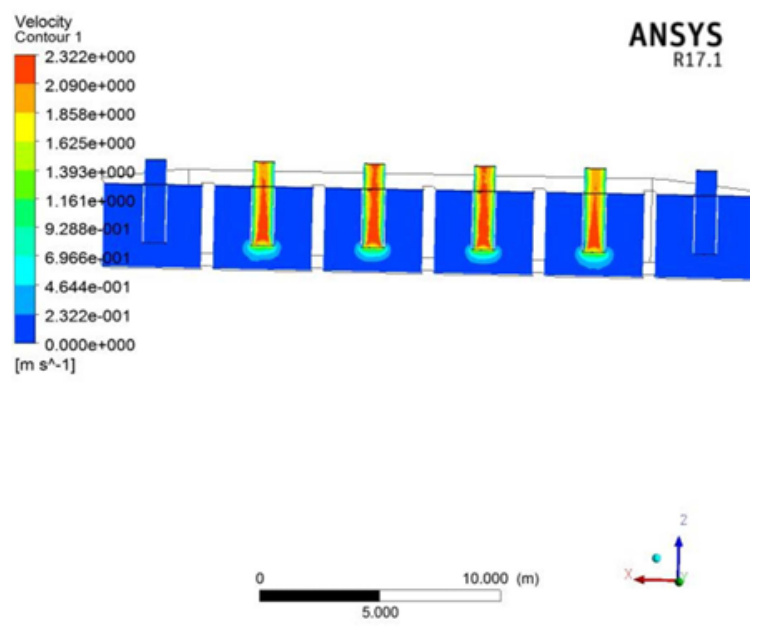

(b) Velocity Contour (distribution)

Figure 8. Case Three: Increase intake pipe length $0.5 \mathrm{~m}$.

\subsection{Case Four: Adding Joint with Length $1 \mathrm{~m}$}

In this case, adding a joint with length $1 \mathrm{~m}$ leading to 
increase the length of submersed part under the water level to become $3 \mathrm{~m}$ and far from intake ground surface by $0.5 \mathrm{~m}$ and four units are operating in this case. The model results show; the vortices appeared as shown in stream line velocity; disturbance is increased slightly at the submersed pipe inlet than cases before Figure (9-a). Velocity inside the suction pipes increased by small changes where it reached to value from $1.935 \mathrm{~m} / \mathrm{sec}$ to $2.419 \mathrm{~m} / \mathrm{sec}$ as shown in velocity contour Figure (9-b).

The results of this case indicate that the flow still uniform in the intake as all cases before, the effect of vortices were appeared in all suction pipe. So, this case mustn't be used because submersed part of pipe near from intake ground surface by $0.5 \mathrm{~m}$. In this case, it was found that the swirl angle returned to increasing where it reached to $8.41^{\circ}$ and still higher than acceptable limit because it became clothier to ground of the intake (As suggested in HI). It was observed that vortices of type 2 as shown in Figure (9-a).

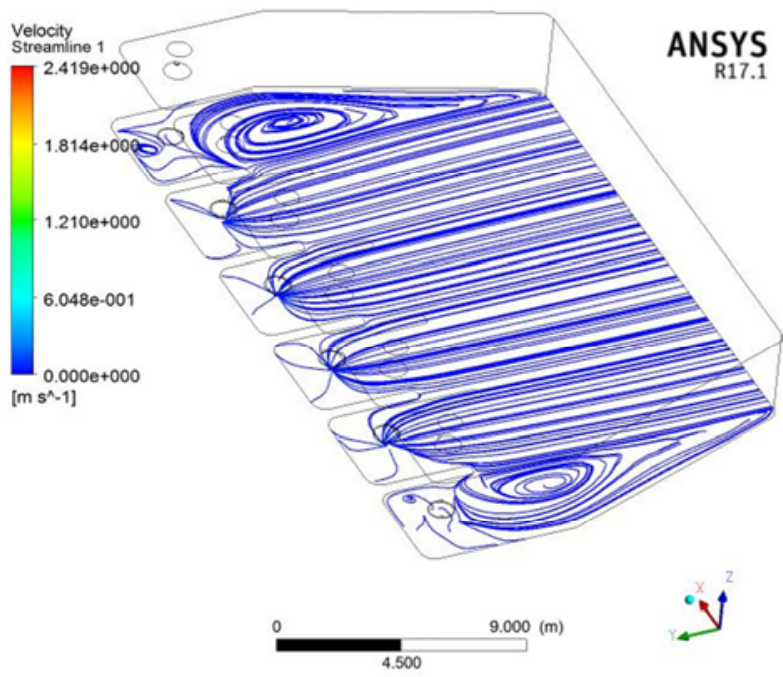

(a) Stream Line Velocity
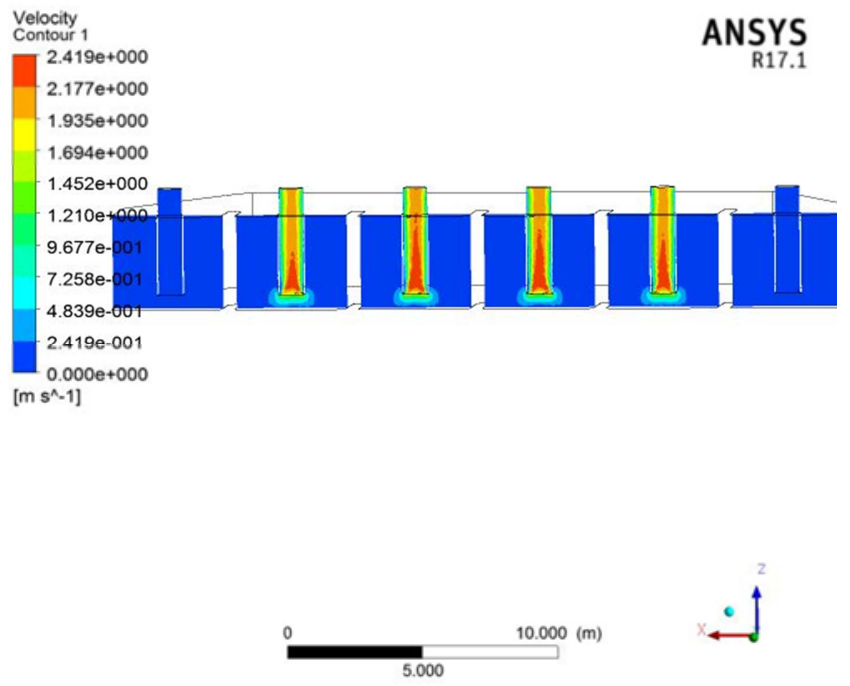

(b) Velocity Contour (distribution)

Figure 9. Case Four: Increase intake pipe length $1 \mathrm{~m}$.

\subsection{Case Five: Adding a Cone Under Bell Mouth with Length $1 \mathrm{~m}$}

In this case, adding a cone with height $1 \mathrm{~m}$ under the bell mouth for each unit in the intake ground surface this means length of submersed part pipe returned to become $2 \mathrm{~m}$ under the water and four units are operating in this case. The model results show; the vortices disappeared from all submersed pipe and disturbance is found, as shown in stream line velocity Figure (10-a). Velocity inside the suction pipes decreased by small changes than cases before where it reached to value from $1.893 \mathrm{~m} / \mathrm{sec}$ to $2.367 \mathrm{~m} / \mathrm{sec}$ as shown in velocity contour Figure (10-b). The results of this case indicate that the flow is not uniform in the intake as all cases before, and the effect of vortices in all submersed pipes become less than all cases before So, this case is better than cases 2,3 , and 4 . In this case, it was found that the swirl angle returned to decreasing where it reached to $7.89^{\circ}$ but still higher than acceptable limit (As suggested in HI). It was observed that vortices of type 2 as shown in Figure (10-a).

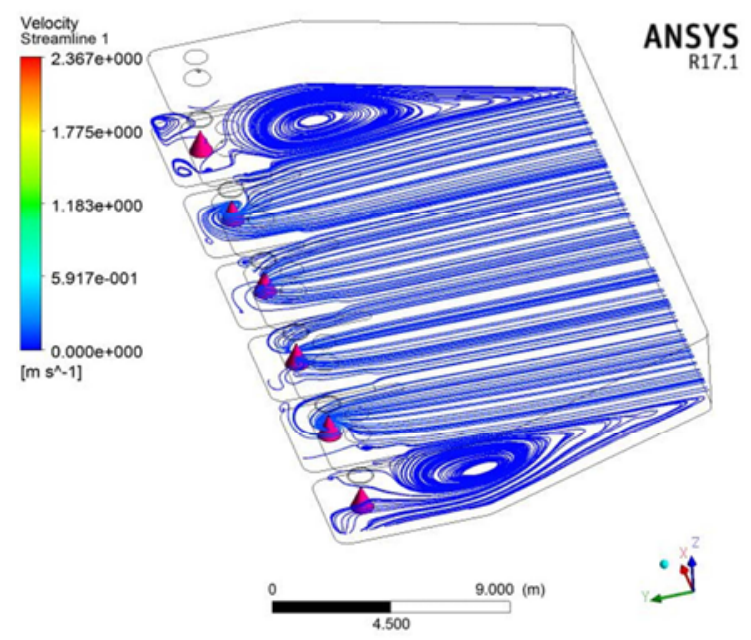

(a) Stream Line Velocity

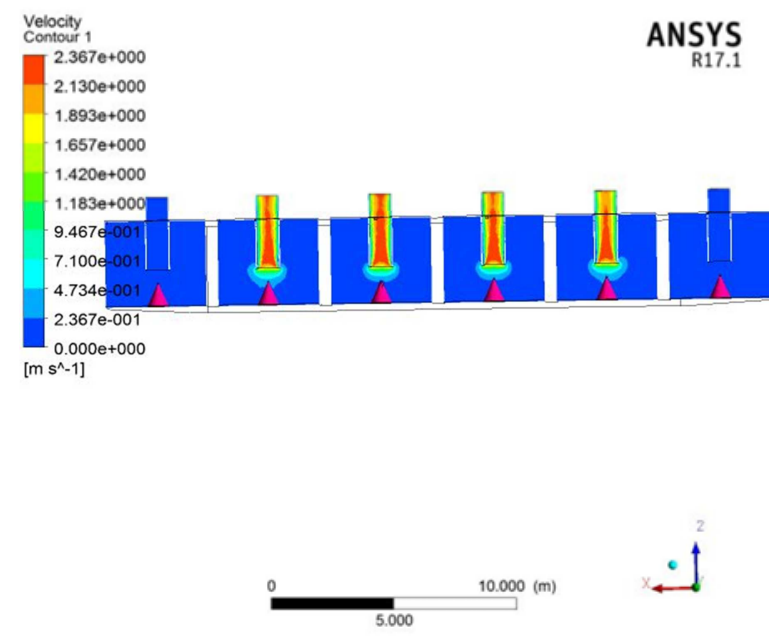

(b) Velocity Contour (distribution)

Figure 10. Case Five: Adding a cone under bell mouth with length $1 \mathrm{~m}$. 


\subsection{Case Six: Adding a Cone Under Bell Mouth with Length $1 \mathrm{~m}$ and $0.5 \mathrm{~m}$ Joint}

In this case, adding a cone under bell mouth with length 1 $\mathrm{m}$ and joint length $0.5 \mathrm{~m}$, this means length of submersed pipe part become $2.5 \mathrm{~m}$ under the water level but with adding cone with height $1 \mathrm{~m}$ in the intake ground surface and four units are operating in this case.

The model results show the vortices disappeared from all submersed part pipes, disturbance in vectors is decreased, and velocity vector has a good distribution as shown in stream line velocity Figure 11-a. Velocity values inside the suction pipes are from $1.946 \mathrm{~m} / \mathrm{sec}$ to $2.433 \mathrm{~m} / \mathrm{sec}$ as shown in velocity contour Figure 11-b.

The results of this case indicate that the flow is uniform and the effect of vortices is disappeared from all submersed pipes. So, this case is better than all cases before.

In this case, it was found that the swirl angle is enhanced where it reached to $5.12^{\circ}$ and this angle become near the standard value (As suggested in $\mathrm{HI}$ ). It was observed that vortices are disappeared as shown in Figure (11-a).

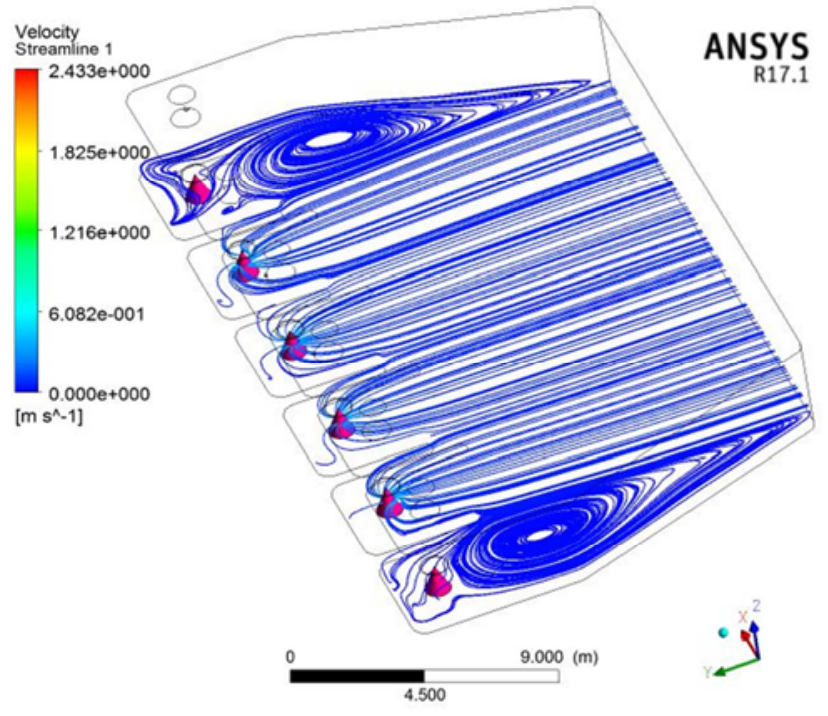

(a) Stream Line Velocity
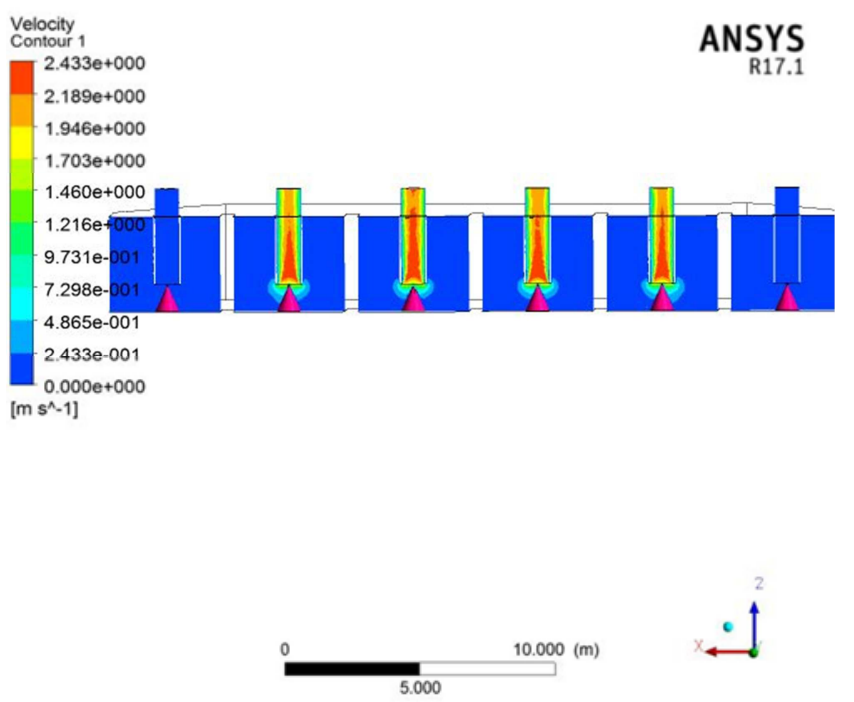

(b) Velocity Contour (distribution)

Figure 11. Case Six: Adding a cone under bell mouth with length $1 \mathrm{~m}$ and 0.5 m joint.

\subsection{Case Seven: Increasing Water Submerging Level to Become $5 m$}

In this case, repeating case 6 but when increasing water submerging Level $30 \%$ then the water level in the intake become $5 \mathrm{~m}$ and the length of submersed pipe part under the water level become $4 \mathrm{~m}$. The model results show the vortices disappeared from all submersed part pipes, disturbance in vectors is decreased, and velocity vector has a good distribution as shown in stream line velocity Figure 12-a. Velocity values inside the suction pipes are from $2.814 \mathrm{~m} / \mathrm{sec}$ to $3.517 \mathrm{~m} / \mathrm{sec}$ as shown in velocity contour Figure 12-b. The results of this case indicate that the flow is still uniform and the effect of vortices also still disappeared from all submersed pipes. So, this case is better than all cases before. In this case, it was found that the swirl angle is enhanced where it reached to $4.66^{\circ}$ and this angle become near the standard value (As suggested in HI) [15]. It was observed that vortices are disappeared as shown in Figure 12-a.

Table 3. Summarizes the results of the seven cases including swirl angle, velocity, and vortex type.

\begin{tabular}{|c|c|c|c|c|c|}
\hline \multicolumn{6}{|c|}{ Summery of the nine cases results } \\
\hline Case No & Specification case & $\begin{array}{l}\text { length of submersed } \\
\text { part }(\mathrm{m})\end{array}$ & $\begin{array}{l}\text { Swirl } \\
\text { angle }\end{array}$ & $\begin{array}{l}\text { Max. Velocity inside } \\
\text { suction pipe } \mathrm{m} / \mathrm{sec}\end{array}$ & Vortex type \\
\hline 1 & Original Case (Optimum design) & 3.5 & $4.85^{\circ}$ & 3.385 & No vortex \\
\hline 2 & $\begin{array}{l}\text { Reduce water submerging Level to become } 3.5 \mathrm{~m} \\
\text { from ground }\end{array}$ & 2.0 & $10.79^{\circ}$ & 2.321 & vortices of type 3 \\
\hline 3 & Add a joint with length $0.5 \mathrm{~m}$ & 2.5 & $7.05^{\circ}$ & 2.322 & vortices of type 1 \\
\hline 4 & Add a joint with length $1 \mathrm{~m}$ & 3.0 & $8.41^{\circ}$ & 2.419 & vortices of type 2 \\
\hline 5 & $\begin{array}{l}\text { Add a cone under bell mouth with height } 1 \mathrm{~m} \text { and } \\
\text { diameter } 0.9 \mathrm{~m}\end{array}$ & $\begin{array}{l}2.0 \\
\text { with Cone }\end{array}$ & $7.89^{\circ}$ & 2.367 & vortices of type 2 \\
\hline 6 & $\begin{array}{l}\text { Add a cone under bell with height } 1 \mathrm{~m} \text {, diameter } \\
0.9 \mathrm{~m} \text { and } 0.5 \mathrm{~m} \text { joint }\end{array}$ & $\begin{array}{l}2.5 \\
\text { with Cone }\end{array}$ & $5.12^{\circ}$ & 2.433 & No vortex \\
\hline 7 & Increasing water submerging level to become $5 \mathrm{~m}$ & 3.5 & $4.66^{\circ}$ & 3.517 & No vortex \\
\hline
\end{tabular}




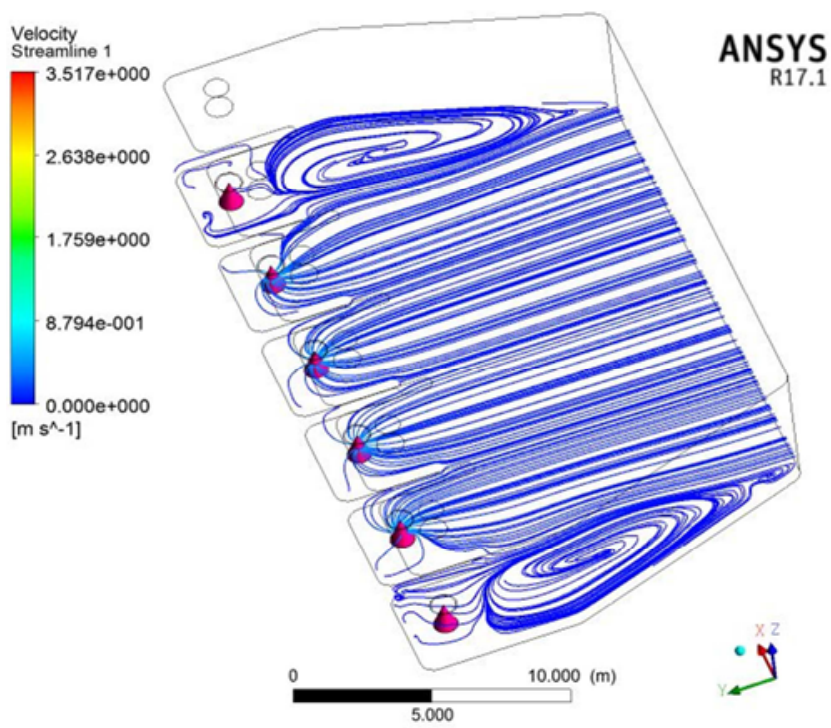

(a) Stream Line Velocity
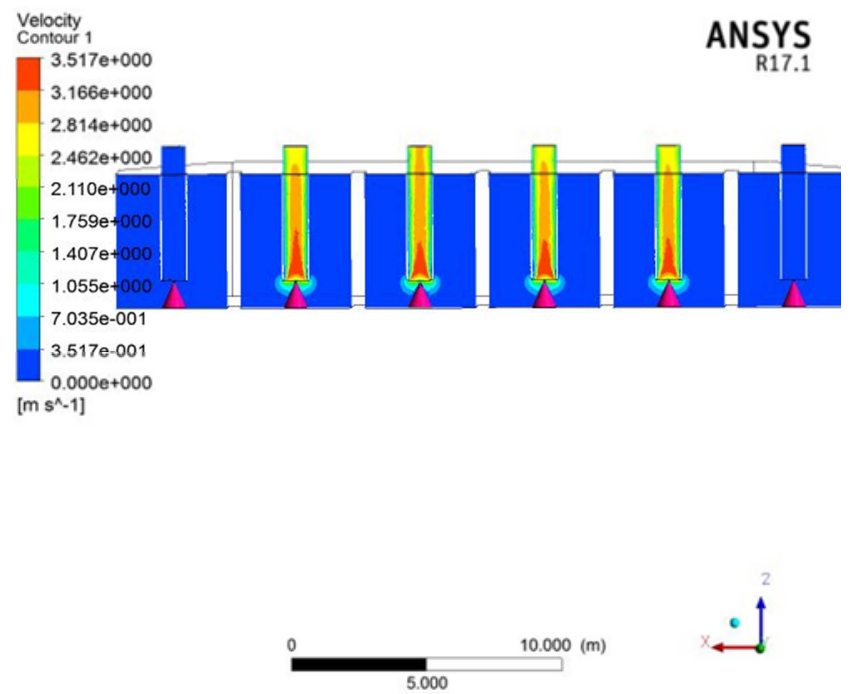

(b) Velocity Contour (distribution)

Figure 12. Case Four: Added a Concrete Wall with steel bars.

\section{Conclusions}

a. Technique of CFD plays an important role for predicting flow characteristics in the suction sumps for purposes of efficient hydraulic performance for both rehabilitation and constructing new pumping stations.

b. The velocity in the submersed part of pipe for low water level conditions decreased about 30\% meaning that flow rate decreased with the same percentage.

c. The research proves that adding a cone down under the bell mouse increases flow rate and reduces vortex, swirling and turbulence at the entrance of the pump, Also, it keeps enhancing hydraulic and dynamic performance of the pumps at the normal operating conditions beyond the winter closure period.

d. This technique can be relied on in the simulation real sumps (multi of scenarios for the shape and movement of water at the entrance to the pump) rather than physical modeling in order to reduce costs and safe time and efforts.

\section{References}

[1] Mohd. Remy Rozainy, I. Abustan, Abdullah. M. Z. and Mohd. Ashraf M. I.," Application of Computational Fluid Dynamics (CFD) in Physical Model of Pump Sump to Predict the Flow Characteristics", ICCBT 08 - D - (07) - pp. 79-90, (2008).

[2] Atefeh Parvaresh Rizil, AbbasRoozbahani1, Salah Kouchakzadeh and Alireza Moridnejad, "Experimental Investigation of Relationship between Sump Flow Pattern and Pumping Energy Consumption", International Symposium on Water Management and Hydraulic Engineering, Ohrid/Macedonia, pp. 131-136, 1-5 Sept. (2009).

[3] S. M. Borghei and A. R. Kabiri-Samani, "Effect of AntiVortex Plates on Critical Submergence at a Vertical Intake", Transaction A: Civil Engineering, Vol. 17, No. 2, pp. 89-95, Sharif University of Technology, April (2010).

[4] Cecilia Lucino, Sergio Liscia y. and Gonzalo Duró, "Vortex Detection in Pump Sumps by Means of CFD", XXIV Latin American Congress on Hydraulics Punta A Del Este, Uruguay, (IAHR), Nov. (2010).

[5] M. A. Younes, "Investigation of Hydraulic Problems in Pumping Station; Case Study" Twelfth International Water Technology Conference, IWTC12, pp. 491-501, Alexandria, Egypt, (2008).

[6] Y X Zhao1, C G Kiml and Y H Lee, "CFD study on flow characteristics of pump sump and performance analysis of the mixed flow pump", 6th International Journal of Pumps and Fans with Compressors and Wind Turbines, 2013.

[7] V. P. Rajendran, S. G. Constantinescu and V. C. Patel, "Experiments on Flow in Model Water pump Intake Sump to Validate a Numerical Model", Proc. of ASME Fluids Engineering Division Summer Meeting FEDSM (Washington, USA, 21-25June 1998), pp. 21-25, 1998.

[8] R. Iwano, T. Shibata, T. Nagahara and T. Okamura, "Numerical Prediction Method of a Submerged Vortex and Its Application to the Flow in Pump Sumps with and without a Baffle Plate", Proc. of the 9th Int. Symp. on Transport Phenomena and Dynamics of Rotating Machinery (Honolulu, Hawaii, USA, 10-14 February 2002)1-6, 2002.

[9] J. W. Choi, Y. D. Choi, C. G. Kim and Y. H. Lee, " Flow Uniformity in a Multi Intake Pump Sump Model", Journal of Mechanical Science and Technology 24 (7) 1389-1400, 2010.

[10] Y. H. Lee, "Establishment of Design Guideline for the Pump Intake Shape using the Result of Model Test", R\&D Report, K-water (in Korean), 2004.

[11] Turbomachinery Society of Japan, "Standard Method for Model Testing the Performance of a Pump Sump", TSJ S002, 2005.

[12] C. G. Kim, J. W. Choi, Y. D. Choi and Y. H. Lee, "A Study on the Effectiveness of an Anti-Vortex Device in the Sump Model by Experiment and CFD", 26th IAHR Symposium on Hydraulic Machinery and Systems, 2012. 
[13] B. E. Launder and D. B. Spalding, "The Numerical Computation of Turbulent Flows", Computer Methods in Applied Mechanics and Engineering, Vol. 3, pp. 269-289, 1974.

[14] ISO 10816-1 (1995), Mechanical Vibration - Evaluation of Machine Vibration by Measurements on Non-Rotating Parts. Part 1, General Guidelines.
[15] American National Standard for Pump Intake Design, ANSI/HI 9.8, 1998.

[16] Hydraulic Institute, "Hydraulic Institute standards for centrifugal, rotary, and reciprocating pumps", $18^{\text {th }}$ edition, Cleveland, Ohio, 1994. 